ANNALES

POLONICI MATHEMATICI

XLV (1985)

\title{
Fonctions algébriques de Grunsky-Shah
}

\author{
par Halina Jondro (Gliwice)
}

\begin{abstract}
The paper deals with the algebraic functions of Grunsky-Shah. We call algebraic function of Grunsky-Shah every function $f \in H(U), f(0)=0, f(z) \neq 0$ for $z \neq 0$, satisfying the equation of the form:

$$
\lambda \log w+\Omega(w)=\hat{\lambda} \log z+x(z),
$$

where the functions $\Omega(w)$ and $\mathscr{X}(z)$ are rational, $\mathscr{X}(z)$ is purely imaginary on the unit circle with 0 and $\propto$ as the only poles of the order $N, \Omega(w)$ is a rational function with 0 as the pole of the order $N$ and satisfying the condition:

$$
\Omega(w)=\overline{-\Omega(-1 / \bar{w})} \quad \text { for every } w .
$$
\end{abstract}

We prove that the class of algebraic functions is dense in the class $\mathscr{K}$.

1. Les propriétés des fonctions algébriques de Grunsky-Shah. Soit $H(U)$ l'espace des fonctions holomorphes dans $U=\{z:|z|<1\}$, avec la convergence uniforme sur des ensembles compacts, $H^{\prime}(U)$ l'espace conjugué, $\mathscr{K}$ la famille des fonctions holomorphes et univalentes dans $U$, de la forme

$$
f(z)=b_{1} z+b_{2} z^{2}+\ldots
$$

et satisfaisant à la condition:

$$
f\left(z_{1}\right) \overline{f\left(z_{2}\right)} \neq-1 \text { pour } z_{1}, z_{2} \in U \text { arbitraires. }
$$

Définissons dans la famille $\mathscr{K}$ la fonctionnelle:

$$
\begin{aligned}
I(f)=\operatorname{Re}\left\{\lambda^{2} \log f^{\prime}(0)+2 \lambda L\left(\log \frac{f(z)}{z}\right)+L^{z}\left(\log \frac{f(z)-f(\zeta)}{z-\zeta}\right)-\right. \\
-\mid L^{2}(\log (1+f(z) \overline{f(\zeta)))\}}
\end{aligned}
$$

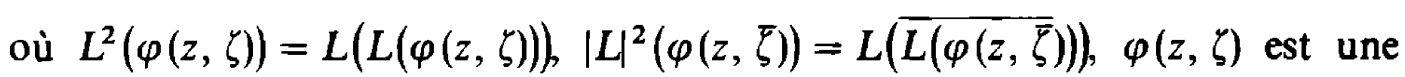
fonction holomorphe dans $U \times U, \hat{\lambda}$ un nombre réel arbitraire.

ThÉORÈme [2]. Si $f$ est la fonction maximale pour la fonctionnelle (3), $f$ satisfait à la relation: 
(4)

$$
\begin{aligned}
\lambda & \log \frac{f(\zeta)}{\zeta}+L\left(\log \frac{f(z)-f(\zeta)}{z-\zeta}\right)-\overline{L(\log (1+\overline{f(\zeta)} f(z)))}+ \\
& +\overline{L(\log (1-\bar{\zeta} z))}=\lambda \log f^{\prime}(0)+L\left(\log \frac{f(z)}{z}\right),
\end{aligned}
$$

et la valeur de la fonctionnelle (3) pour $f$ admet la forme suivante:

$$
\operatorname{Re} I(f)=I(f)=-|L|^{2}(\log (1-\bar{\zeta} z)) \text {. }
$$

Définissons la fonctionnele $L$ de (3) de la manière suivante: $L(1)=0$, $L\left(z^{n}\right)=\lambda_{n}$, pour $n=1, \ldots, N, L\left(z^{m}\right)=0$ pour $n>N$, où $\lambda_{n}$ sont des nombres complexés arbitraires. Par exemple, une fonctionnelle qui satisfait à ces conditions est la fonctionnelle linéaire $L=L(h)$, dépendant seulement du nombre fini des coefficients de la fonction $h$. Dans ce cas, on peut écrire l'équation de la fonction extrémale (4) sous une forme plus concrète, en exprimant les fonctions $\log \frac{f(z)-f(\zeta)}{z-\zeta}$ et $\log (1+f(z) \overline{f(\zeta)})$ par les polynômes de Faber. Ainsi, en utilisant la relation connue

$$
\log (1-t f(z))=-\sum_{m=1}^{\infty} \frac{1}{m}\left(F_{m}(t)-m a_{m 0}\right) z^{m}
$$

où $\sum_{m=0}^{\infty} a_{m 0} z^{m}=\log f(z) / z$ et $F_{m}(t)$ désigne le polynôme de Faber de degré $m$ pour la fonction $1 / f(z)$, nous obtenons, en posant successivement $1 / f(\zeta)$ et $-\overline{f(\zeta)}$ au lieu de $t$ dans les relations (5)

$$
\log \frac{f(z)-f(\zeta)}{z-\zeta}=-\sum_{m=1}^{\infty} \frac{1}{m}\left(F_{m}\left(\frac{1}{f(\zeta)}\right)-m a_{m 0}\right) z^{m}-\log (\zeta-z)+\log f(\zeta)
$$

et

$$
\log (1+f(z) \overline{f(\zeta)})=-\sum_{m=1}^{\infty} \frac{1}{m}\left(F_{m}(\overline{-f(\zeta)})-m a_{m 0}\right) z^{m}
$$

En tenant encore compte du fait que pour $z$ suffisamment proches de 0

$$
\log (\zeta-z)=\log \zeta-\sum_{k=1}^{\infty} \frac{1}{k} \cdot \frac{1}{\zeta^{k}} z^{k} \quad \text { et } \quad \log (1-\bar{\zeta} z)=-\sum_{k=1}^{\infty} \frac{1}{k} \zeta^{k} z^{k}
$$

et du fait que $\operatorname{Re}\left(\lambda \log f^{\prime}(0)+L(\log (f(z) / z))\right)=0$ ((11) dans [2]), nous constatons que la fonction extrémale $w=f(\zeta)$ satisfait à l'équation fonctionnelle de la forme:

$$
\lambda \log w-\sum_{m=1}^{N} \frac{1}{m}\left(F_{m}(1 / w)-\overline{F_{m}(-\bar{w})}\right)=\lambda \log \zeta-\sum_{k=1}^{N}\left(\frac{1}{k}\left(\frac{\lambda_{k}}{\zeta^{k}}-\bar{\lambda}_{k} \zeta^{k}\right)\right)+C
$$


où $C$ est une constante imaginaire. L'équation (6) est un cas particulier d'une équation plus générale, notamment de l'équation de la forme:

$$
\lambda \log w+\Omega(w)=\lambda \log z+\mathscr{X}(z),
$$

où $\Omega(w)$ et $\mathscr{X}(z)$ sont des fonctions rationnelles, $\mathscr{X}(z)$ est une fonction imaginaire sur la circonférence $\partial U$ admettant des pôles seulement aux points 0 et $\infty$. Désignons l'ordre de ces pôles par $N$ (vu la symétrie $\mathscr{X}(z)$ par rapport à $\partial U$, l'ordre de ces deux pôles doit être le même). $\Omega(w)$ est une fonction rationnelle possédant, entre autres, le pôle 0 d'ordre $N$ et satisfisant à la condition

$$
\Omega(w)=\overline{-\Omega(-1 / \bar{w})} \quad \text { pour tout } w .
$$

Nous allons prouver le théorème suivant:

ThÉOREm 1. Si $w=f(z), f \in H(U), f(0)=0, f(z) \neq 0$ pour $z \neq 0$, est une fonction qui satisfait à léquation (7) dans $U$, et si $\log f(z) / z$ est une certaine branche de $\log f(z) / z$, alors

$1^{\circ} f$ se prolonge dune manière continue sur le cercle unité, fermé,

$2^{\circ} f \in \mathscr{K}$,

$3^{\circ}$ le bord $\partial D, D=f(U)$, se confond avec $\Gamma=f(\partial U)$. Ce bord est composé des points $w$ pour lesquels $\operatorname{Re}\{\lambda \log w+\Omega(w)\}=0$.

Démonstration. La démonstration se compose de 3 parties qui correspondent aux propriétés annoncées.

ad $1^{\circ}$. Pour prouver la première propriété il suffit de démontrer que pour chaque suite de points $\left\{z_{j}\right\}, z_{j} \rightarrow z_{0},\left|z_{j}\right|<1,\left|z_{0}\right|=1$, la suite $f\left(z_{j}\right) \rightarrow w_{0}$ dépend seulement de $z_{0}$. Considérons l'équation

$$
i \log w+\Omega(w)=\not x\left(z_{0}\right)+\lambda \log z_{0} .
$$

Soient $w_{h}$ des racines différentes de l'équation (9). Nous allons démontrer que le nombre des solutions de l'équation (9) est fini. Supposons le contraire. Soit $\left\{w_{h}\right\}$ la suite des solutions de l'équation ci-dessus. Seuls les pôles de la fonction $\Omega(w)$ peuvent être des points d'accumulation de la suite $\left\{w_{h}\right.$ '.

Supposons que $w_{h} \rightarrow w^{*} \neq \infty$ et soit $w^{*}$ un pôle d'ordre $L$ de la fonction $\Omega\left(w^{*}\right.$ peut être aussi 0 , donc en même temps un point singulier de la fonction $\log w$ ). De plus multiplions les deux membres de l'égalité

$$
\lambda \log w_{h}+\Omega\left(w_{h}\right)=\mathscr{X}\left(z_{0}\right)+\lambda \cdot \log z_{0} \quad \operatorname{par}\left(w_{h}-w^{*}\right)^{L} .
$$

Passant à la limite avec $h \rightarrow \infty$, nous obtenous la conclusion qu'un coefficient de $\left(w_{h}-w^{*}\right)^{-L}$ dans la série de Laurent de la fonction $\Omega(w)$ doit être égal à zéro, en contradiction avec la definition de $L$ comme ordre du pôle $w^{*}$. De même nous pouvons prouver que $\infty$ ne peut être un point d'accumulation de la suite $\left.; w_{h}\right\}$. Ainsi nous avons démontré que le nombre des solutions de l'équation (9) est fini; désignons - le par $R$. 
Soit à présent $\varepsilon>0$ un nombre arbitraire, suffisamment petit pour que tous les cercles $K\left(w_{h}, \varepsilon\right), h=1, \ldots, R$, pris avec leurs circonférences soient disjoints. Soit $U_{0}$ un voisinage du point $z_{0}$ tel que pour $z \in U_{0} \cap U, z$ arbitraire, toutes les racines de l'équation (7) appartiennent à l'ensemble $S=\bigcup_{h} K\left(w_{h}, \varepsilon\right)$. Mais pour chaque $z \in U_{0} \cap U$ les nombres $f(z)$ sont les racines de l'équation (7).

D'autre part, $f\left(U_{0} \cap U\right)$ est un ensemble connexe, donc il existe $h_{0}$ tel que $f\left(U_{0} \cap U\right) \subset K\left(w_{h_{0}}, \varepsilon\right)$. Donc si $z_{j} \in U_{0} \cap U$, alors

$$
f\left(z_{j}\right) \in K\left(w_{h_{0}}, \varepsilon\right) \text {. }
$$

Puisque $\varepsilon>0$ est un nombre arbitraire et que la condition (10) est remplie, il résulte que $f\left(z_{j}\right) \rightarrow w_{h_{0}}$, et, en vertu de la définition $w_{h_{0}}$, que $w_{h_{0}}$ dépend seulement de $z_{0}$. En posant donc $w_{0}=w_{h_{n}}$ nous obtenons que la suite $f\left(z_{j}\right)$ $\rightarrow w_{0}$, et la propriété $1^{\circ}$ est ainsi prouvée.

ad $2^{\circ}$. Considérons la fonction $h(z)=-1 / \overline{f(\bar{z})}$. C'est une fonction méromorphe dans $U$, avec un seul pôle $z=0$. Posons $D^{\prime}=h(U), \Gamma_{1}=h(\partial U)$. Après un calcul facile, en utilisant l'identité (8), nous constatons que la fonction $w=h(z)$ satisfait à l'équation

$$
\lambda \log (w z)+\Omega(w)=-\overline{\mathscr{X}(\bar{z})}+\lambda \log (-1) \quad \text { dans } U,
$$

où $\log (h(z) z)$ est une certaine branche du logarithme de la fonction $z h(z)$ dans $U$. En vertu de $1^{\circ} f(z)$ et $h(z)$ se prolongent d'une façon continue sur le cercle $U$, d'où' nous déduisons que $f$ et $h$ satisfont respectivement aux équations (7) et (11) dans $\bar{U}$. Comme les seconds membres des équations (7) et (11) sont imaginaires sur la circonférence $\partial U$, on a $\operatorname{Re}\{\lambda \log w+\Omega(w)\}$ $=0$, sur $\Gamma$, ainsi que sur $\Gamma_{1}$. En outre, puisque les seconds membres des équations (7) et (11) sont bornés sur la circonférence $\hat{\partial} U$, les courbes $\Gamma$ et $\Gamma_{1}$ ne passent ni par 0 ni par $\infty$ (ni d'ailleurs par d'autres pôles de la fonction $\Omega$ ), donc en particulier elles sont bornées. On voit aussi tout de suite que $\partial D \subset \Gamma$ et $\partial D^{\prime} \subset \Gamma_{1}$, car $f(z)$ et $h(z)$ sont méromorphes dans $U$. Ainsi $\partial D$ et $\partial D^{\prime}$ sont des ensembles bornés. Ni zéro, ni aucun des pôles de la fonction $\Omega(w)$ ne leur appartiennent pas. Puisque $\partial D$ est borné et la fonction $f$ est holomorphe dans $U$, il résulte que $D$ est aussi borné; le domaine $D^{\prime}$ n'est évidemment pas borné, car il contient $\infty$.

On peut prouver, de plus, que les continus $\Gamma$ et $\Gamma_{1}$ ne coupent pas respectivement les domaines $D$ et $\dot{D}^{\prime}$. Remarquons d'abord que $D$ et $D^{\prime}$ ne contiennent aucun pôle de la fonction $\Omega(w)$, que $0 \in D$ et $\infty \in D^{\prime}$. Supposons ensuite que $\Gamma$ coupe le domaine $D$, c'est-à-dire que $D \backslash \Gamma$ n'est pas connexe. Alors, outre la composante $S_{0}$ contenant le point 0 , il existerait une seconde composante $S$, qui est bornée comme ensemble compris dans l'ensemble borné $D$. Le bord de cette composante est contenu évidemment dans $\partial D \cup \Gamma$, donc dans $\Gamma$. Conformément à ce qui précède la fonction $\lambda \log w+\Omega(w)$ 
aurait sur le bord du domaine $S$ une partie réelle nulle ce qui n'est pas possible, car chaque branche de cette fonction est holomorphe dans $S$ ( $S$ ne contient pas de pôles de la fonction $\Omega(w)$ ) et cette fonction n'est pas constante. De même on peut prouver que $\Gamma_{1}$ ne coupe pas $D^{\prime}$. Si $\Gamma_{1}$ coupait $D^{\prime}$, alors outre la composante non bornée $S_{0}, \infty \in S_{0}$, il existerait encore une composante bornée $S$ telle que $0, \infty \notin S$, ce qui n'est pas possible pour la même raison que ci-dessus.

A présent nous allons prouver que $f(U) \cap h(U)=D \cap D^{\prime}=\emptyset$. Supposons en effet, que $D \cap D^{\prime} \neq \emptyset$. L'ensemble $D \cap D^{\prime}$ est certainement ouvert et ne contient pas 0 et $\infty$, car $\infty \notin D$ et $0 \notin D^{\prime}$. Le bord de cet ensemble est compris dans $\partial D \cup \partial D^{\prime}$, donc dans $\Gamma \cup \Gamma_{1}$. En considérant la partie réelle de la fonction $\lambda \log w+\Omega(w)$, nous obtenons une contradiction.

Pour achever la démonstration de la deuxième partie, nous devons prouver encore que la fonction $f(z)$ est univalente. Cette démonstration est pareille à celle du travail [1].

ad $3^{\circ}$. Des considérations précédentes nous savons que $\partial D \subset \Gamma$. Il suffit alors de montrer que inversement, $\partial D \supset \Gamma$. Supposons que $\Gamma \notin \partial D$. Comme $\Gamma \subset \bar{D}$ il en résulte que l'ensemble $\Gamma$ aurait un point commun $w_{0}$ avec $D$. Alors il y aurait des points $\zeta_{0} \in \partial U$ et $z_{0} \in U$ tels que, $f\left(\zeta_{0}\right)=f\left(z_{0}\right)=w_{0}$, ce qui n'est pas possible, car la fonction $f(z)$ est univalente.

De faits précédents il résulte que $\hat{\lambda} \log w+\Omega(w)$ est imaginaire sur $\partial D$.

Nous appellerons fonction algébrique de Grunsky-Shah chaque fonction $f \in H(U), f(0)=0, f(z) \neq 0$ pour $z \neq 0$, qui satisfait à l'équation du type (7), où $\Omega(w)$ et $\mathscr{X}(z)$ sont les fonctions mentionnées au début de ce paragraphe.

2. La densité de la classe des fonctions algébriques dans la classe $\mathscr{K}$. Soit $f \in \mathscr{K}$. Posons $D=f(U), D^{\prime}=h(U)$, où $h(z)=-1 / \overline{f(\bar{z})}$. Evidemment $D \cap D^{\prime}$ $=\emptyset$. Considérons la fonction $z=f^{-1}(w)$ inverse de $f(z)$ dans $D$ et posons

$$
F(w)= \begin{cases}f^{-1}(w), & w \in D, \\ \overline{f^{-1}(-1 / \bar{w}),} & w \in D^{\prime} .\end{cases}
$$

C'est une fonction holomorphe dans l'ensemble ouvert $D \cup D^{\prime}$. Soit $r$ un nombre arbitraire, $0<r<1$, et soient $D_{r}=f(\{|z|<r\}), D_{r}^{\prime}=h(\{|z|<r\})$. Evidemment $\bar{D}_{r} \cup \bar{D}_{r}^{\prime} \subset D \cup D^{\prime}$. En vertu du théorème de Runge pour $\varepsilon>0$ arbitraire, $0<\varepsilon<\frac{1}{2}(1-r)$, il existe une fonction rationnelle $r_{\varepsilon}(w)$ telle que

$$
\left|\frac{1}{2} F(w)-r_{\varepsilon}(w)\right|<\varepsilon \quad \text { pour } w \in D_{r} \cup \bar{D}_{r}^{\prime},
$$

et possédant des pôles uniquement dans $C \backslash\left(D \cup D^{\prime}\right)$.

On peut poser que $r_{e}(0)=r_{\varepsilon}(\infty)=0$. En effet, dans le cas contraire, transformons la surface $(w)$ à l'aide de l'homographie

$$
\omega=H(w)=\frac{w-a}{w-b}
$$


où $a \neq b, b \in C \backslash\left(\bar{D}_{r} \cup \bar{D}_{r}^{\prime}\right)$, $a$ est arbitraire. $H\left(\bar{D}_{r}\right)$ et $H\left(\bar{D}_{r}^{\prime}\right)$ sont des domaines fermés et bornés, $H(0)=a / b, \quad H(\infty)=1, \quad w=H^{-1}(\omega)=(b \omega-a) /(\omega-1)$. Considérons la fonction

$$
\Phi(\omega)=F\left(H^{-1}(\omega)\right) /(\omega-a / b)(\omega-1) .
$$

En vertu de $F(0)=F(\infty)=0$, la fonction $\Phi(\omega)$ est holomorphe dans $H(D) \cup H\left(D^{\prime}\right)$. Vu le théorème de Runge pour $\varepsilon^{\prime}>0$ arbitraire, il existe une fonction rationnelle $\varrho(\omega)$, avec des pôles situés dans $C \backslash\left(H(D) \cup H\left(D^{\prime}\right)\right.$ ), donc différents de $a / b$ et 1 , telle que

alors

$$
\left|\frac{1}{2} \Phi(\omega)-\varrho(\omega)\right|<\varepsilon^{\prime} \quad \text { pour } \omega \in H\left(\bar{D}_{r}\right) \cup H\left(\bar{D}_{r}^{\prime}\right) ;
$$

$$
\left|\frac{1}{2} F\left(H^{-1}(\omega)\right)-(\omega-a / b)(\omega-1) \varrho(\omega)\right|<\varepsilon|\omega-a / b||\omega-1| \leqslant \varepsilon^{\prime} \cdot M,
$$

pour

$$
\omega \in H\left(\bar{D}_{r}\right) \cup H\left(\bar{D}_{r}^{\prime}\right), \quad \text { où } \quad M=\sup _{\boldsymbol{H}\left(\bar{D}_{r}\right) \cup H\left(D_{r}^{\prime}\right)}|\omega-a / b||\omega-1| .
$$

Posant maintenant $\omega=H(w)$ et $\varepsilon^{\prime}=\varepsilon / M$ dans (12), nous obtenons

$$
\left|\frac{1}{2} F(w)-(H(w)-a / b)(H(w)-1) \varrho(H(w))\right|<\varepsilon \quad \text { dans } \bar{D}_{r} \cup \bar{D}_{r}^{\prime} .
$$

La fonction

$$
r_{\varepsilon}(w)=(H(w)-a / b)(H(w)-1) \varrho(H(w))
$$

est déjà telle que $r_{\varepsilon}(0)=r_{\varepsilon}(\infty)=0$, et elle est holomorphe dans $D \cup D^{\prime}$, car le pôle $b$ ne lui appartient pas, et les pôles de la fonction $\varrho(\omega)$ n'appartiennent pas à $H(D) \cup H\left(D^{\prime}\right)$.

Posons maintenant

$$
Q_{\varepsilon}(w)=r_{\varepsilon}(w)+\overline{r_{\varepsilon}(-1 / \bar{w})},
$$

c'est une fonction rationnelle, $Q_{\varepsilon}(0)=0$, elle est holomorphe dans $D \cup D^{\prime}$, et enfin

$$
\left|F(w)-Q_{\varepsilon}(w)\right|<2 \varepsilon \quad \text { dans } \bar{D}_{r} \cup \bar{D}_{r}^{\prime} .
$$

En effet, si $w \in \bar{D}_{r}$, alors

$$
\begin{aligned}
\left|F(w)-Q_{\varepsilon}(w)\right| & \leqslant\left|\frac{1}{2} F(w)-r_{\varepsilon}(w)\right|+\left|\frac{1}{2} F(w)-\overline{r_{\varepsilon}(-1 / \bar{w})}\right| \\
& =\left|\frac{1}{2} f^{-1}(w)-r_{\varepsilon}(w)\right|+\left|\frac{1}{2} f^{-1}(w)-\overline{r_{\varepsilon}(-1 / \bar{w})}\right|
\end{aligned}
$$

et comme

$$
\left|\frac{1}{2} f^{-1}(w)-r_{\varepsilon}(w)\right|<\varepsilon \quad \text { pour } w \in \bar{D}_{r}
$$

et

$$
\left|\frac{1}{2} \overline{f^{-1}(-1 / \bar{\omega})}-r_{\varepsilon}(\omega)\right|<\varepsilon \quad \text { pour } \omega \in \bar{D}_{r}^{\prime},
$$


alors en posant dans la dernière inégalité $\omega=-1 / \bar{w}, w \in \bar{D}_{r}$, nous obtenons $\left|\frac{1}{2} f^{-1}(w)-\overline{r_{\varepsilon}(-1 / \bar{w})}\right|<\varepsilon$, de même que $w \in \bar{D}_{r}^{\prime}$, alors (13) est remplie. On a aussi

$$
Q_{\varepsilon}(w)=\overline{Q_{\varepsilon}(-1 / \bar{w})},
$$

et pour $\varepsilon$ suffisamment petit $Q_{\varepsilon}(w)$ est univalente dans $\bar{D}_{r}$, et aussi dans $\bar{D}_{r}^{\prime}$ donc en particulier $Q_{\varepsilon}(\omega) \neq 0$ pour $\omega \neq 0, \omega \in \bar{D}_{r}$. Puisque $f^{-1}\left(\partial D_{r}\right)=\{|z|$ $=r\}$, en vertu de (13) $Q_{\varepsilon}\left(\partial D_{r}\right) \subset\left\{r-2 \varepsilon<|z|<r+2 \varepsilon_{\}}\right.$, et il résulte que $\{|z|$ $=r-2 \varepsilon\} \subset Q_{\varepsilon}\left(D_{r}\right)$. Posons. $\Gamma_{r \varepsilon}=Q_{\varepsilon}^{-1}(\{|z|=r-2 \varepsilon\})$. On voit que $Q_{\varepsilon}\left(\Gamma_{r \varepsilon}\right)$ $=\{|z|=r-2 \varepsilon\}$, et encore en vertu de la convergence uniforme $Q_{\varepsilon}^{-1}(z) \rightarrow f(z)$ avec $\varepsilon \rightarrow 0$ dans le cercle $U_{r}=\{|z| \leqslant r\}$, les courbes $\Gamma_{r \varepsilon}$ sont convergentes vers la courbe $\partial D_{r}$ au sens de Fréchet, c'est-a-dire au sens de la convergence uniforme des descriptions ${ }^{\circ}$ paramétriques.

En effet, soit $Z \subset U_{r}$ un ensemble arbitraire, fermé et soit $\varrho$ $=\operatorname{dist}(Z, C \backslash U)$. Si $2 \varepsilon<\varrho$, alors $Z \subset Q_{\varepsilon}\left(D_{r}\right)$, car $U_{r-2 \varepsilon} \subset Q_{\varepsilon}\left(D_{r}\right)$, et $Z \subset \bar{U}_{r-\rho} \subset U_{r-2 \varepsilon}$. Soit $\eta>0$ arbitraire. De la continuité uniforme de la fonction $f$ dans $Z$ nous déduisons qu'il existe $\sigma>0$ tel que si $z_{1}, z_{2} \in Z$ et $\left|z_{1}-z_{2}\right|<\sigma$, alors $\left|f\left(z_{1}\right)-f\left(z_{2}\right)\right|<\eta$. Mais, en vertu de (13), $\left|Q_{\varepsilon}(w)-f^{-1}(w)\right|$ $<2 \varepsilon$ pour chaque $w \in \bar{D}_{r}$. En posant $w=Q_{\varepsilon}^{-1}(z), z \in Z$, dans la dernière inégalité, nous obtenons l'inégalité $\left|z-f^{-1}\left(Q_{\varepsilon}^{-1}(z)\right)\right|<2 \varepsilon$. Alors si $\varepsilon<\frac{1}{2} \sigma$, $\left|f(z)-Q_{\varepsilon}^{-1}(z)\right|<\eta$, ce qui prouve la convergence uniforme de $Q_{\varepsilon}^{-1}(z)$ vers $f(z)$ dans $U$. Evidemment $\Gamma_{r \varepsilon} \subset D_{r}$, donc l'intérieur de la courbe $\Gamma_{r \varepsilon}$ appartient à $D_{r}$ en vertu du fait que $D_{r}$ est connexe. En désignant cet intérieur par $G_{r \varepsilon}$, nous constatons que $0 \in G_{r \varepsilon}$, et que $G_{r \varepsilon}$ converge avec $\varepsilon \rightarrow 0$ vers $D_{r}$, au sens de la convergence vers le noyau.

La fonction rationnelle $Q_{\varepsilon}(w)$ est holomorphe dans $\bar{G}_{r \varepsilon}$ et elle s'annule dans $\bar{G}_{r \varepsilon}$ seuleument au point $w=0$.

Enfin posons

$$
\Omega_{\varepsilon}(w)=\frac{i}{\frac{1}{r-2 \varepsilon} Q_{\varepsilon}(w)}+\frac{i}{(r-2 \varepsilon) Q_{\varepsilon}(w)}
$$

C'est une fonction rationnelle qui possède un pôle unique dans $G_{r \varepsilon}$ au point $w=0$, en plus, en vertu de (14) et (15), la relation suivante a lieu

$$
\Omega_{\varepsilon}(w)=-\overline{\Omega_{\varepsilon}(-1 / \bar{w})},
$$

et on a

$$
\operatorname{Re} \Omega_{\varepsilon}(w)=0 \quad \text { pour } w \in \Gamma_{r \varepsilon} .
$$

A présent on peut démontrer le théorème suivant:

ThÉOREMe 2. La classe des fonctions algébriques de Grunsky-Shah est dense dans la classe $\mathscr{K}$. 
Démonstration. Soit $f \in \mathscr{K}$ et $\hat{f}_{r}(z)=f(r z), 0<r<1$. Evidemment $\hat{f}_{r} \in \mathscr{X}$ et $\hat{f}_{r}(z) \rightarrow f(z)$ où $r \rightarrow 1^{-}$presque uniformément dans $U$. Si $Z \subset U$ est un ensemble arbitraire compact, alors pour $\eta>0$ arbitraire il existe $r_{0}$, $0<r_{0}<1$, tel que pour chaque $r_{0}<r<1$ et pour chaque $z \in Z$

$$
\left|\hat{f}_{r}(z)-f(z)\right|<\eta \text {. }
$$

Maintenant soit $r_{1}, r_{0}<r_{1}<1$, arbitraire, mais fixé. Soit $G_{r_{1} \varepsilon}$ la famille de domaines dicrite ci-dessus, construite pour le domainc $D_{r_{1}}$. Soit la fonction $\hat{f}_{\varepsilon}(z), \hat{f}_{\varepsilon}(0)=0$, qui représente $U$ sur $G_{r_{1} \varepsilon}$. En vertu de la convergence $G_{r_{1} \varepsilon}$ à $D_{r_{1}}$ où $s \rightarrow 0$ au sens de la convergence vers le noyau, $\hat{f}_{\varepsilon}(z) \rightarrow \hat{f}_{r_{1}}(z)$ presque uniformément dans $U$, d'où il existe un $\varepsilon_{0}$ tel que pour $0<\varepsilon<\varepsilon_{0}$ et pour chaque $z \in Z$

$$
\left|\hat{f}_{\varepsilon}(z)-f_{r_{1}}(z)\right|<\eta \text {. }
$$

$\mathrm{Vu}$ (18) et (19) nous concluons que pour $0<\varepsilon<\varepsilon_{0}$ et pour chaque $z \in Z$

$$
\left|\hat{f_{\varepsilon}}(z)-f(z)\right|<2 \eta,
$$

ce qui prouve la possibilité d'approcher presque uniformément la fonction $f$ par les fonctions $\hat{f}_{\varepsilon}$.

D'autre part, $\hat{f}_{\varepsilon}$ sont des fonctions algébriques de Grunsky-Shah. En effet, la composition $\Omega_{\varepsilon}\left(\hat{f}_{\varepsilon}(z)\right), z \in U$, est une fonction méromorphe dans $U$ avec un pôle seulement au point $z=0$. En outre, vu le théorème de Carathéodory-Osgood, $\hat{f}_{\varepsilon}$ étant une fonction qui représente le cercle $U$ sur le domaine $G_{r \varepsilon}$, borné par la courbe de Jordan $\Gamma_{r \varepsilon}$, elle se prolonge d'une façon continue sur le cercle fermé $U$. en représentant d'une façon homéomorphe $\bar{U}$ sur $\bar{G}_{r \varepsilon}=G_{r \varepsilon} \cup \Gamma_{r \varepsilon}$. La fonction $\Omega_{\varepsilon}\left(\hat{f}_{\varepsilon}(z)\right)$ se prolonge donc d'une façon continue sur $\bar{U}$, et en vertu de (17) $\operatorname{Re} \Omega_{\varepsilon}\left(\hat{f}_{\varepsilon}(z)\right)=0$ pour $z \in \partial U$. Vu le principe de la symétrie de Schwarz la fonction $\Omega_{\varepsilon}\left(\hat{f}_{\varepsilon}(z)\right)$ se prolonge comme fonction rationnelle avec les seuls pôles $z=0$ et $z=x$ sur tout le plan, restant imaginaire sur $\partial U$. En désignant la fonction ainsi prolongée par $\mathscr{X}_{\varepsilon}(z)$, nous concluons que la fonction $\hat{f}_{\varepsilon}$ est une fonction algébrique de Grunsky-Shah, ce qui achève la démonstration du théorème.

\section{Travaux cités}

[1] Z. Charzyński, J. Śladkowska-Zahorska, Application de la méthode des fonctions alyébriques à la représentation conforme, Dissertationes Math. 195 (1982). 160.

[2] H. Jondro, Les inégalités du type de Grunsky pour fonctions de la classe $\mathscr{X}$, Ann. Polon. Math., ce volume, 43-53. 